\title{
Exciting Graphene Surface Plasmon Polaritons through Light and Sound Interplay
}

\author{
Mohamed Farhat, ${ }^{1, *}$ Sébastien Guenneau, ${ }^{2}$ and Hakan Bağcl ${ }^{1}$ \\ ${ }^{1}$ Division of Computer, Electrical, and Mathematical Sciences and Engineering, 4700 King Abdullah University of Science \\ and Technology, Thuwal 23955-6900, Saudi Arabia \\ ${ }^{2}$ Aix-Marseille Université, CNRS, Centrale Marseille, Institut Fresnel, F-13013 Marseille, France
}

(Received 2 June 2013; published 5 December 2013)

\begin{abstract}
We propose a concept that allows for efficient excitation of surface plasmon spolaritons (SPPs) on a thin graphene sheet located on a substrate by an incident electromagnetic field. Elastic vibrations of the sheet, which are generated by a flexural wave, act as a grating that enables the electromagnetic field to couple to propagating graphene SPPs. This scheme permits fast on-off switching of the SPPs and dynamic tuning of their excitation frequency by adjusting the vibration frequency (grating period). Potential applications include single molecule detection and enhanced control of SPP trajectories via surface wave patterning of graphene metasurfaces. Analytical calculations and numerical experiments demonstrate the practical applicability of the proposed concept.
\end{abstract}

Graphene is a honeycomb crystal of carbon atoms, which was first isolated by Novoselov et al. in 2004 [1]. Investigations of its electrical and mechanical properties have subsequently confirmed that it is a revolutionary material with promising applications in various fields of applied physics and engineering [2]. Graphene's ultrahigh carrier mobility, excellent thermal stability, and unusual carrier-density-dependent surface conductivity [3] make it a promising building block for various and ubiquitous devices operating at terahertz $(\mathrm{THz})$ and infrared (IR) frequencies [4]. The high absorption of white light by an atomically thin sheet of graphene led to the design of various photonic devices for light modulation or photodetection [5,6]. More recently, graphene has been shown to support propagation of guided surface electromagnetic waves at its interface with a dielectric, so-called graphene surface plasmon polaritons (GSPPs). GSPPs have longer propagation lengths compared to metallic SPPs [7]. Additionally, their characteristics can be tuned through chemical doping or by applying an external gate voltage to the graphene sheet. These unusual properties combined with the need for tunable photonic or plasmonic devices working over a broad band of frequencies have fueled research on graphene. Consequently, it has recently been used in building $\mathrm{THz}$ antennas [8-10], plasmonic waveguides [11], transformation-optics devices [12,13], perfect absorbers [14,15], and sensors [16].

However, to further exploit all potential avenues opened by graphene's unprecedented interaction with electromagnetic fields, methods for efficiently exciting GSPPs must be developed. This is a challenging task due to the strong mismatch between wave numbers in graphene and free space at THz and IR frequencies [17]. To this end, several approaches, including use of nano- and microribbons [7] or more classical schemes such as the Otto-Kretschmann configurations [18], have been proposed. Additionally, it has been demonstrated in $[19,20]$ that GSPPs can be excited using highly localized fields that are generated by metal near-field probes acting as nanoantennas. However, these methods are limited by excitation of highly localized GSPPs.

In this Letter, we propose a concept that exploits periodic diffraction gratings generated on a thin graphene sheet, which would enable efficient excitation of propagating GSSPs by an incident electromagnetic field at $\mathrm{THz}$ and IR frequencies [21]. It is well known that periodic corrugations on metallic surfaces lead to a matching condition necessary to generate SPPs for various frequencies and angles of arrival of the exciting electromagnetic field. Similar ideas apply to excitation of propagating GSSPs [22]. Recent studies have analyzed the effect of periodicity in the permittivity of the dielectric substrate supporting a thin planar graphene sheet but not the periodicity in the topography of the sheet itself [23].

Our proposal, which exploits surface elastic waves to produce gratings from a thin graphene sheet, is described schematically in Fig. 1. A sheet of carbon atoms of thickness $\delta$ is located on a substrate with permittivity $\varepsilon_{d}=2.25$ and excited from one end by a mechanical vibrator. Graphene's Young modulus $E$ exceeds by far that of all known materials (including steel) [24] and $\delta$ is very small $(\delta<1 \mathrm{~nm})$. These make it possible to model elastic vibrations of the graphene sheet in the transversal direction by the scalar biharmonic equation $\left(\Delta^{2}-\beta_{b}^{4}+\kappa / D\right) W=q$, where $W, q, \beta_{b}$, and $D$ represent the displacement field, source of vibrations (a concentrated point or line force), the flexural wave number, and the flexural rigidity, respectively [25]. The coefficient $\kappa$ accounts for the stiffness of the substrate, which is modeled as a Winkler foundation exerting an additional reaction force $-\kappa W$ on the thin plate [26]. This implies that the above biharmonic equation can be recast into a simpler one defining a new parameter 


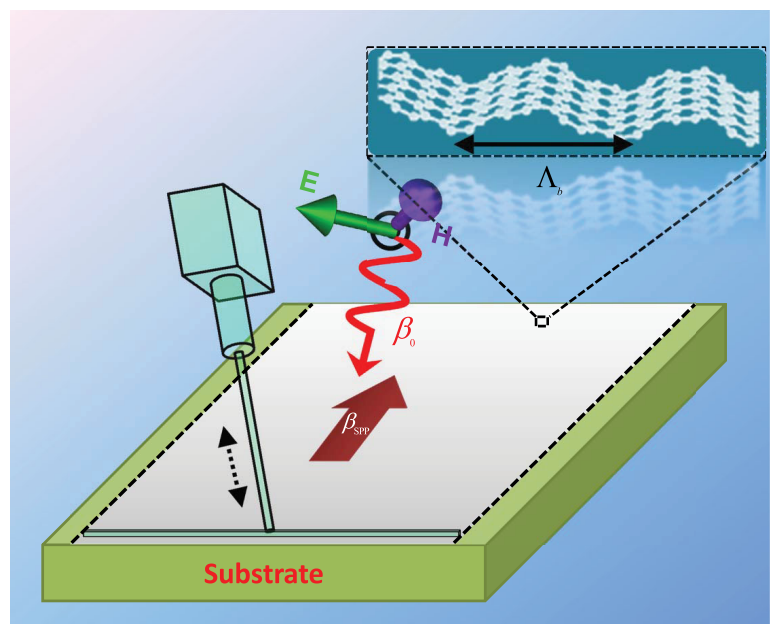

FIG. 1 (color online). Geometry under investigation: a thin graphene sheet on top of a substrate is simultaneously excited by a biharmonic plane wave with wave number $\beta_{b}$ and an electromagnetic field with wave number $\beta_{0}$. The inset shows the graphene patterned as a sinusoidal grating. The thick red arrow indicates propagating GSPPs with wave number $\beta_{\text {SPP }} \gg \beta_{0}$.

$\tilde{\beta}_{b}^{4}=\beta_{b}^{4}-\kappa / D:\left(\Delta^{2}-\tilde{\beta}_{b}^{4}\right) W=q$. Elastic waves that satisfy this biharmonic equation without the source (i.e., $q=0$ ) and the reaction force from the substrate (i.e., $\kappa=0)$ are the acoustic phonon modes of the honeycomb crystal and their propagation constant satisfies the dispersion relation $\beta_{b}^{2}=\omega_{b} \sqrt{\rho \delta / D}$. Here $\omega_{b}$ is the vibration frequency, $\rho$ is the density, and the flexural rigidity is given by $D=E \delta^{3} /\left[12\left(1-\nu^{2}\right)\right]$, where $\nu$ is the Poisson coefficient $[26,27]$. The elastic wave generated on the graphene sheet has a wavelength $\Lambda_{b}=2 \pi / \operatorname{Re}\left(\tilde{\beta}_{b}\right)$ (inset of Fig. 1). Figure 2(a) plots the normalized dispersion relation of the
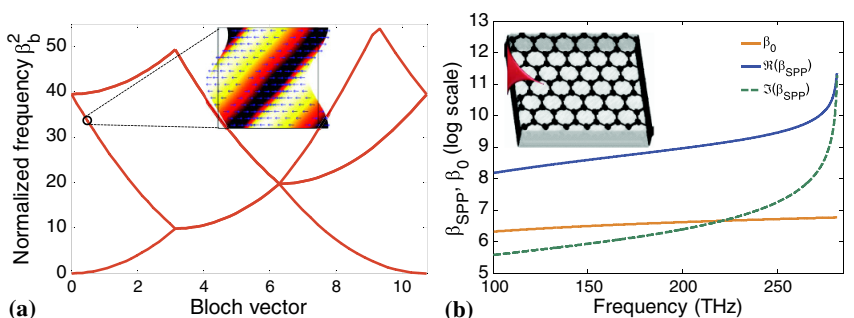

FIG. 2 (color online). (a) Dispersion relation of the flexural mode. The horizontal axis is the projection of the Bloch vector on the boundary of the first irreducible Brillouin zone ГХM. The vertical axis is the square of the flexural wave number $\beta_{b}^{2}=$ $\omega_{b} \sqrt{\rho \delta / D}$ with $\delta=0.3 \mathrm{~nm}, \rho=2300 \mathrm{~kg} / \mathrm{m}^{3}$, and $D=$ $2.34 \times 10^{-18} \mathrm{~Pa} \mathrm{~m}^{3}$. The inset shows a snapshot of the vertical displacement field $W$ in the unit cell and the arrows represent the energy flow of elastic power. (b) Dispersion relation of the GSPP with $E_{F}=0.7 \mathrm{eV}$ and dispersion relation of an electromagnetic wave in surrounding space. The inset shows the geometry of the thin graphene sheet lying on top of a dielectric substrate (silicon) of permittivity $\varepsilon_{d}=2.25$. bending modes of the graphene sheet along the first Brillouin zone $\Gamma X M$ for $\delta=0.3 \mathrm{~nm}, \rho=2300 \mathrm{~kg} / \mathrm{m}^{3}$, $E=10^{12} \mathrm{~Pa}, \nu=0.2$, and $D=2.34 \times 10^{-18} \mathrm{~Pa} \mathrm{~m}^{3}$ [24]. The inset shows the profile of $W$ within the unit cell (the arrows showing the flow of elastic energy). From the dispersion relation in Fig. 2(a), it can be seen that $\Lambda_{b}=$ $250 \mathrm{~nm}$ when the flexural wave frequency $\omega_{b} /(2 \pi)$ is around $195 \mathrm{MHz}$.

For electromagnetic analysis at $\mathrm{THz}$ and IR frequencies, this flexural wave of a graphene sheet can accurately be modeled as a static grating with period $\Lambda_{b}$ and is utilized to couple incident electromagnetic fields to propagating GSPPs. Electromagnetic wave interactions on the graphene grating are analyzed using the finite-elementmethod (FEM) [28]. It should be noted here that quantum effects are ignored in the electromagnetic simulations owing to the lateral dimensions of our structure (Fig. 1): It was shown in [29] that plasmons generated on structures with lateral dimensions larger than $10 \mathrm{~nm}$ could be treated using the classical electromagnetic theory. Graphene's complex dielectric permittivity is modeled using $\varepsilon_{V, G} \approx 1+j \sigma_{\text {intra }} / \delta \varepsilon_{0} \omega \approx-\omega_{G, \mathrm{p}}^{2} / \omega\left(\omega+j \tau^{-1}\right)$ $[9,17]$. Here, $\sigma_{\text {intra }}$ is the intraband conductivity, $\omega_{G, \mathrm{p}}=$ $\sqrt{e^{2} E_{F} / \delta \varepsilon_{0} \pi \hbar^{2}}$ is the effective plasma frequency, $E_{F}$ is the Fermi energy, $\tau$ is the intrinsic relaxation time and assumed to follow the relation $\tau=\mu E_{F} /\left(e v_{F}^{2}\right)$ (that accounts for variation of loss with Fermi energy), $e$ is the electronic charge, and $\hbar$ is the reduced Planck constant, $v_{F}=c / 300$ the Fermi velocity ( $c$ is the speed of light) and $\mu=15000 \mathrm{~cm}^{2} \mathrm{~V}^{-1} \mathrm{~s}^{-1}$ is the measured dc mobility [30,31]. As pointed out in recent papers [9,12], when $\operatorname{Re}\left(\varepsilon_{V, G}\right)<0$, graphene sustains the propagation of transverse magnetic (TM) plasmon modes that possess very high confinement. The dispersion relation associated with these modes is given by $\beta_{\mathrm{SPP}}=$ $\beta_{0} \sqrt{1+\left[\left(1+\varepsilon_{d}\right) /\left(\delta \eta_{0} \varepsilon_{0} \varepsilon_{V, G} \omega\right)\right]^{2}}$, where $\beta_{\mathrm{SPP}}$ is the GSPP's wave number, $\beta_{0}$ and $\eta_{0}$ are the wave number and impedance in free space, respectively, and $\varepsilon_{d}$ is the permittivity of the dielectric substrate. Figure 2(b) compares $\beta_{\text {SPP }}$ computed for $E_{F}=0.7 \mathrm{eV}$ to $\beta_{0}$ in logarithmic scale. It is clear that there is a very strong mismatch between these wave numbers; they are separated with nearly 2 orders of magnitude. The salient consequence is that it is virtually impossible to excite GSPPs directly by electromagnetic fields originating in free space. In this work, this limitation is overcome by bending the graphene sheet using flexural waves as described next.

The period of the grating, $\Lambda_{b}$, (generated by the bending flexural wave), $\beta_{0}$ and $\beta_{\text {SPP }}$ satisfy the relation

$$
\operatorname{Re}\left[\beta_{\mathrm{SPP}}\right]-\beta_{0} \sin \theta=\tilde{\beta}_{b}=2 \pi N / \Lambda_{b}
$$

which is obtained through phase matching at the interface between the graphene and free space. Here, $\theta$ is the angle 
of arrival of the incident field and $N$ is an integer that denotes the grating mode order. Inserting the expression of $\beta_{\text {SPP }}$ into Eq. (1) and exploiting the fact that $\beta_{\text {SPP }} \gg \beta_{0}$ yields the following equation in frequency $\omega$

$$
\omega^{2}=\frac{e^{2} E_{F}}{\hbar^{2} \varepsilon_{0}\left(1+\varepsilon_{d}\right)}\left(\frac{2 N}{\Lambda_{b}}+\frac{\omega \sin \theta}{\pi c}\right) .
$$

This nonlinear equation has two solutions. Let the positive solution be represented by $\omega_{\text {res }}$; clearly $\omega_{\text {res }}$ is the frequency at which the GSPP can be excited by the incident electromagnetic field. It is expected that at these frequencies, the absorption spectrum will have peaks since the incident field's energy is coupled to the GSSP and is propagated on the surface of the graphene.

To demonstrate that GSSPs are indeed generated at frequency $\omega_{\text {res }}$, numerical electromagnetic simulations of the setup shown in Fig. 1 excited by a normally incident plane wave $(\theta=0)$ with magnetic field along the $z$ direction $\left(H_{z}\right)$ are carried out. For these simulations, $E_{F}=1 \mathrm{eV}, \omega_{b} /(2 \pi)=195 \mathrm{MHz}$, and $\Lambda_{b}=250 \mathrm{~nm}$. Figure 3(a) plots the absorption, transmission, and
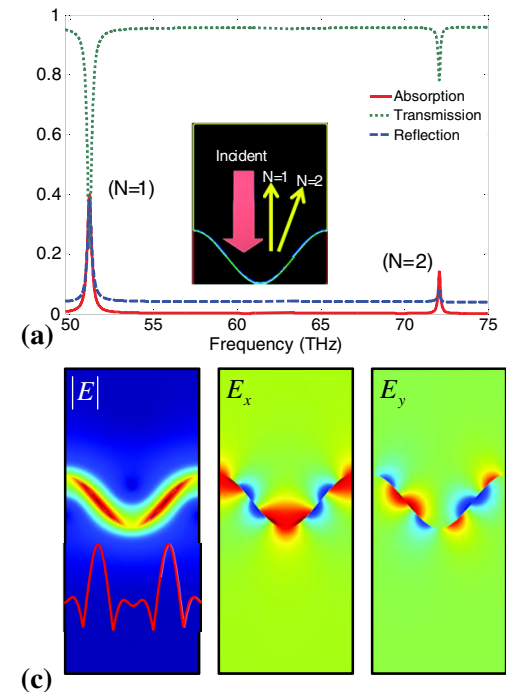

(b)
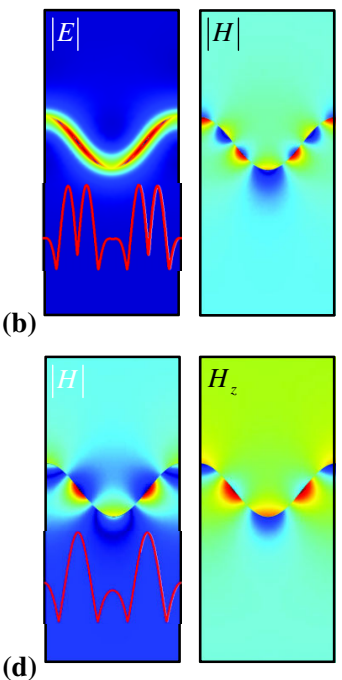

FIG. 3 (color online). (a) Absorption, transmission, and reflection spectra of the graphene sheet with $\delta=0.3 \mathrm{~nm}$ and $E_{F}=$ $1 \mathrm{eV}$, which is vibrated at flexural frequency $\omega_{b} /(2 \pi)=$ $175 \mathrm{MHz}\left(\Lambda_{b}=250 \mathrm{~nm}\right)$. The inset shows the direction of electromagnetic excitation $(\theta=0)$ and the resulting reflected waves (thin arrows). Note that only the specular reflection was taken into account since the period of the grating $\Lambda_{b}=250 \mathrm{~nm}$ is very small compared to wavelength of the incident electromagnetic field $\lambda_{0}=7 \mu \mathrm{m}$. (b) Electric and magnetic field norms in the unit cell for the $N=2$ mode, where the induced GSPP's wave number is $4 \pi / \Lambda_{b}$. Different components of (c) the electric field and (d) the magnetic field demonstrate the resonances associated with the $N=1$ mode, where the induced GSPP's wave number is $2 \pi / \Lambda_{b}$. The periodicity in the profile of the electric field norm is plotted along the grating's upper boundary and it shows the order of each corresponding grating mode. reflection spectra. Two peaks occurring at 51 and $72 \mathrm{THz}$ are clearly identified. An analytical solution of Eq. (2) for $N=1$ and $N=2$ indeed confirms that the vibrating graphene sheet sustains GSPPs propagating at these frequencies. The first one is the fundamental mode $(N=1)$ and is dominant with absorption reaching $45 \%$; it is also associated with the excitation of the GSPP with $\operatorname{Re}\left(\tilde{\beta}_{\mathrm{SPP}}\right)=$ $2 \pi / \Lambda_{b}$. The absorption of the second mode $(N=2)$ reaches almost 20\% (two and a half times less); it is associated with the GSPP with $\operatorname{Re}\left(\tilde{\beta}_{\mathrm{SPP}}\right)=4 \pi / \Lambda_{b}$. Figure 3 (b) plots the distribution of electric and magnetic fields norms for the $N=2$ mode and Figs. 3(c) and 3(d) plot field norms and several field components for the $N=1$ mode. These figures clearly show the structure of these modes: the norm of the electric field plotted along the grating sinusoidal boundary shows a $2 \pi$ periodicity for the fundamental mode and $4 \pi$ for the other one. A strong enhancement of electromagnetic fields is observed particularly for the fundamental mode where the absorption reaches $45 \%$. Figure 3(a) shows that without the GSPPs being excited, the absorption is less than $1 \%$ (at frequencies excluding 51 and $72 \mathrm{THz}$ ). This means that the GSPPs are responsible for absorption of about $45 \%$ of the incident electromagnetic field's energy. It is worth mentioning at this stage that this value is in actuality the upper limit for a thin symmetric layer as pointed out in [14]. One possible way of increasing further the absorption by the graphene gratings is to add a reflecting layer below our design; this would result in the cancellation of the transmission channel and at the GSPP resonances, all of the incident energy will be absorbed by the graphene layer: this is the so-called perfect absorber studied in depth recently $[14,15]$. An increase in the absorption of graphene discs and nanoribbons at certain frequencies has also been observed in earlier studies $[14,15]$. Nonetheless, it should be mentioned here that this is a completely different physical mechanism than the one described here. The increase in the absorption of graphene discs and ribbons is due to the excitation of the localized GSPPs unlike the propagating ones induced on the vibrating graphene sheet as described here.

It is clear from Eq. (2) that $\omega_{\text {res }}$ is a function of $\Lambda_{b}, E_{F}$, and $\theta . \Lambda_{b}$ and $E_{F}$ can be thought of as dynamically tunable device design parameters: $\Lambda_{b}$ can be controlled through the elastic dispersion relation of the graphene sheet by varying $\omega_{b}$, and $E_{F}$ can be controlled by applying an external gate voltage to the graphene sheet. Additionally, $\theta$ is also easy to vary. Electromagnetic simulations are carried out to demonstrate the effect of these parameters on the response of the vibrating graphene sheet for three sets of simulations. Simulation set (i): $\Lambda_{b}=250 \mathrm{~nm}, \theta=0$, and $E_{F}$ is varied between 0.5 and 1.1. eV. Figures 4(a) and 4(b) plot the absorption spectra for various values of $E_{F}$. It is clearly shown that $\omega_{\text {res }}$ can be tuned between $35-55 \mathrm{THz}$ for the $N=1$ mode [Fig. 4(a)] and $50-75 \mathrm{THz}$ for the $N=2$ mode [Fig. 4(b)] by varying $E_{F}$. This broad tuning of the 

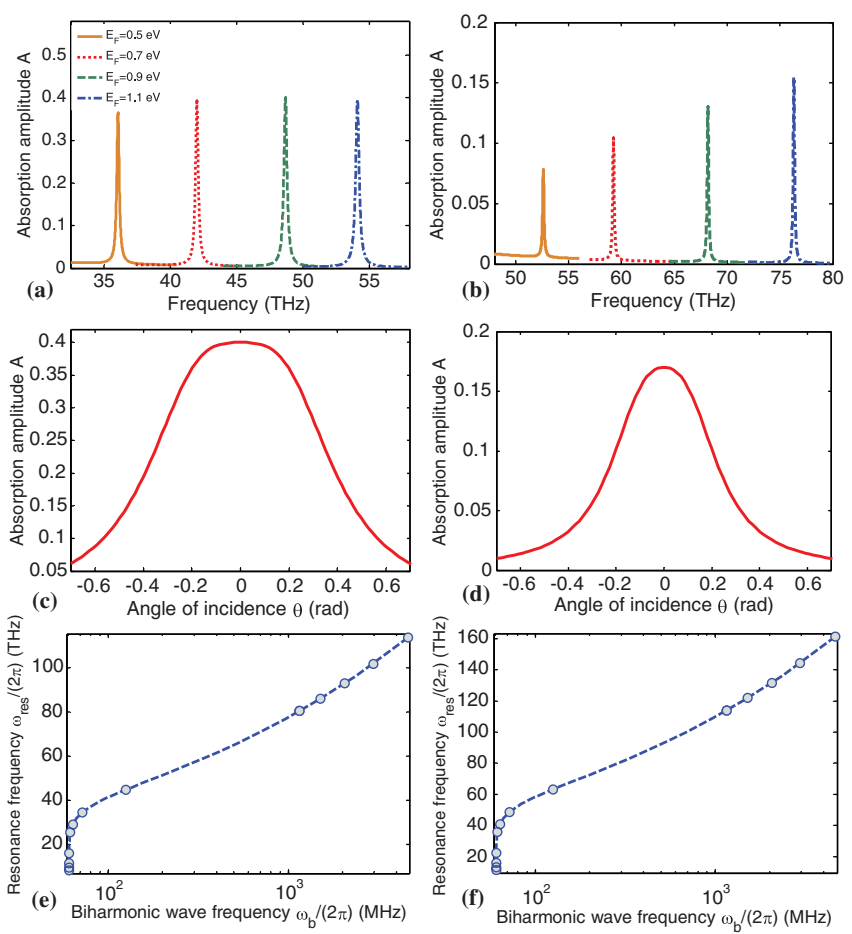

FIG. 4 (color online). Absorption spectra of the vibrating graphene sheet when $\Lambda_{b}=250 \mathrm{~nm}$ and $\theta=0$ for four values of $E_{F}$ (a) for the $N=1$ mode and (b) for the $N=2$ mode. Absorption amplitude vs $\theta$ when $E_{F}=1 \mathrm{eV}$ and $\Lambda_{b}=250 \mathrm{~nm}$ (c) at frequency $51 \mathrm{THz}$ for the $N=1$ mode and (d) at frequency $72.1 \mathrm{THz}$ for the $N=2$ mode. GSPP resonance frequency $\omega_{\text {res }} /(2 \pi)$ vs flexural frequency $\omega_{b} /(2 \pi)$ when $E_{F}=1 \mathrm{eV}$ and $\theta=0$ (e) for the $N=1$ mode and (f) for the $N=2$ mode. The continuous curves are obtained by solving Eq. (2) for $\omega_{\text {res }}$ with given $\omega_{b}$ or $\Lambda_{b}$. Dots represent frequencies $\omega_{\text {res }}$ obtained via electromagnetic simulations. Note the good agreement.

resonance frequency could be understood from Eq. (2), where for normal incidence, the resonance frequency is of the form $\omega_{\text {res }} \approx \sqrt{N E_{F} / \Lambda_{b}}$. This relation explains also the larger tunability of the second mode and the factor $\sqrt{2}$ between resonance frequencies of both modes at same Fermi energy. The propagation length normalized to the GSPP wavelength is defined as the ratio $\operatorname{Re}\left(\tilde{\beta}_{\mathrm{SPP}}\right) / \operatorname{Im}\left(\tilde{\beta}_{\mathrm{SPP}}\right)[12]$ and is equal to $120,201,296$, and 402 for $E_{F} 0.5,0.7,0.9$, and $1.1 \mathrm{eV}$, respectively. As expected, it is higher for larger Fermi levels (lower intrinsic losses). Simulation set (ii): $E_{F}=1 \mathrm{eV}, \Lambda_{b}=250 \mathrm{~nm}$, the frequency is $51 \mathrm{THz}$ for the $N=1$ mode and $72 \mathrm{THz}$ for the $N=2$ mode, and $\theta$ is varied from $-\pi / 4$ to $\pi / 4$. Figures 4(c) and 4(d) plot the absorption vs $\theta$. Figure 4(c) shows that the amplitude of absorption for the $N=1$ mode reaches very high values approaching $45 \%$ in a symmetrical manner. For $\theta=0$, its maximum is achieved; this means that coupling between the incident field and the GSPPs is maximized due to matching in their respective wave numbers. The effect can be considered as robust to the variations in $\theta$ since the absorption's full width at half maximum (FWHM) is around $\pi / 4$. Figure 4 (d) shows that the amplitude of absorption for the $N=2$ mode behaves quite differently: It has also a maximum for $\theta=0$, but it is more sensitive to the variations in $\theta$ and the FWHM is around $\pi / 8$. Simulation set (iii): $E_{F}=1 \mathrm{eV}, \theta=0$, and $\omega_{b} /(2 \pi)$ is varied between $60 \mathrm{MHz}$ and $46 \mathrm{GHz}$ (corresponding to periods of the grating $\Lambda_{b}$ ranging from $50 \mathrm{~nm}$ to $10 \mu \mathrm{m})$. Higher values of $\Lambda_{b}$ will result in convergence of $\omega_{b}$ to $\sqrt{\rho h \kappa} / D$ because of the presence of the Winkler foundation term $\kappa / D$, where $\kappa=10^{11} \mathrm{~N} \mathrm{~m}^{-1}$ [32]. Figures 4(e) and 4(f) plot the GSPP resonance frequency $\omega_{\text {res }} /(2 \pi)$ vs biharmonic wave natural frequency $\omega_{b} /(2 \pi)$. The continuous curves are obtained by solving Eq. (2) for $\omega_{\text {res }}$ given $\omega_{b}\left(\right.$ or $\left.\Lambda_{b}\right)$. Dots represent $\omega_{\text {res }}$ obtained via electromagnetic simulations. The agreement is excellent. These two figures also demonstrate the potential of our approach since it is shown that $\omega_{\text {res }}$ could be dynamically tuned from 10 to $120 \mathrm{THz}$ and from 10 to $160 \mathrm{THz}$ for the grating modes with $N=1$ and $N=2$, respectively.

In this Letter we have proposed to couple elastic vibrations of very thin graphene sheets to light in order to generate highly confined GSPPs. Our numerical and theoretical simulations demonstrate the possibility to achieve reconfigurable gratings that could have highly tunable and fast response to light. By stopping the elastic vibration, it is indeed feasible to turn off rapidly the graphene plate. This could be a significant step towards the integration of graphene plasmonics devices into realistic and multiple applications and prompt further advances in the emerging field of transformational plasmonics as well [33]. Finally, our concept permits us not only to excite GSPPs but also to tune them by means of their physical (Fermi energy) and geometrical (period of the grating) properties.

S. G. wishes to thank the European Research Council for an ERC starting Grant. M. F. would like to acknowledge fruitful discussions about the theory of gratings with Shakeeb Bin Hasan. The authors also would like to acknowledge insightful comments from anonymous referees, which greatly helped to improve the quality of this Letter.

Note added.-Schiefele et al. [34] recently reported a different technique to excite graphene plasmons through electrically generated surface acoustic waves.

*mohamed.farhat@kaust.edu.sa

[1] K. S. Novoselov, A. K. Geim, S. V. Morozov, D. Jiang, Y. Zhang, S. V. Dubonos, I. V. Grigorieva, and A. A. Firsov, Science 306, 666 (2004).

[2] European Graphene Flagship (www.grapheneflagship.eu); K. Kupferschmidt, Science 339, 497 (2013).

[3] A. H. C. Neto, F. Guinea, N. M. R. Peres, K. S. Novoselov, and A. Geim, Rev. Mod. Phys. 81, 109 (2009).

[4] A. N. Grigorenko, M. Polini, and K. S. Novoselov, Nat. Photonics 6, 749 (2012). 
[5] M. Liu, X. Yin, E. Ulin-Avila, B. Geng, T. Zentgraf, L. Ju, F. Wang, and X. Zhang, Nature (London) 474, 64 (2011).

[6] F. Xia, T. Mueller, Y. M. Lin, A. Valdes-Garcia, and P. Avouris, Nat. Nanotechnol. 4, 839 (2009).

[7] L. Ju, B. Geng, J. Horng, C. Girit, M. Martin, Z. Hao, H. A. Bechtel, X. Liang, A. Zettl, Y. R. Shen, and F. Wang, Nat. Nanotechnol. 6, 630 (2011); A. Y. Nikitin, F. Guinea, F. J. Garcia-Vidal, and L. Martin-Moreno, Phys. Rev. B 84, 161407 (2011).

[8] K. Y. Shin, J. Y. Hong, and J. Jang, Adv. Mater. 23, 2113 (2011).

[9] F. H. L. Koppens, D. E. Chang, and F. J. G. de Abajo, Nano Lett. 11, 3370 (2011).

[10] M. Tamagnone, J. Gomez-Diaz, J. Mosig, and J. Perruisseau-Carrier, J. Appl. Phys. 112, 114915 (2012); R. Filter, M. Farhat, M. Stieglich, R. Alaee, C. Rockstuhl, and F. Lederer, Opt. Express 21, 3737 (2013).

[11] J. Christensen, A. Manjavacas, S. Thongrattanasiri, F. H. L. Koppens, and F. J.G. de Abajo, ACS Nano 6, 431 (2012).

[12] A. Vakil and N. Engheta, Science 332, 1291 (2011).

[13] P. Y. Chen and A. Alù, ACS Nano 5, 5855 (2011); M. Farhat, C. Rockstuhl, and H. Bagci, Opt. Express 21, 12592 (2013).

[14] S. Thongrattanasiri, F. H. L. Koppens, and F. J. García de Abajo, Phys. Rev. Lett. 108, 047401 (2012).

[15] R. Alaee, M. Farhat, C. Rockstuhl, and F. Lederer, Opt. Express 20, 28017 (2012).

[16] R. Lv et al., Sci. Rep. 2, 586 (2012); M. Amin, M. Farhat, and H. Bagci, Sci. Rep. 3, 2105 (2013).

[17] M. Jablan, H. Buljan, and M. Soljacic, Phys. Rev. B 80, 245435 (2009).

[18] Y. V. Bludov, M. I. Vasilevskiy, and N. M. R. Peres, Europhys. Lett. 92, 68001 (2010).

[19] J. Chen et al., Nature (London) 487, 77 (2012); Z. Fei et al., Nature (London) 487, 82 (2012).
[20] A. Y. Nikitin, F. Guinea, F. J. Garcia-Vidal, and L. MartinMoreno, Phys. Rev. B 84, 195446 (2011).

[21] K. V. Sreekanth, S. Zeng, J. Shang, K. T. Yong, and T. Yu, Sci. Rep. 2, 737 (2012).

[22] A. Ferreira and N. M. R. Peres, Phys. Rev. B 86, 205401 (2012); T. M. Slipchenko, M. L. Nesterov, L. MartinMoreno, and A. Yu. Nikitin, arXiv:1307.0310.

[23] W. Gao, J. Shu, C. Qiu, and Q. Xu, ACS Nano 6, 7806 (2012).

[24] J. W. Jiang, J. S. Wang, and B. Li, Phys. Rev. B 80, 113405 (2009); S. S. Gupta and R. C. Batra, J. Comput. Theor. Nanosci. 7, 2151 (2010).

[25] M. Farhat, S. Guenneau, S. Enoch, A. B. Movchan, and G. G. Petursson, Appl. Phys. Lett. 96, 081909 (2010); Europhys. Lett. 91, 54003 (2010).

[26] S. Timoshenko, Theory of Plates and Shells (McGrawHill, New York, 1940).

[27] F. Graff, Wave Motion in Elastic Solids (Dover, New York, 1975).

[28] http://www.comsol.com.

[29] S. Thongrattanasiri, A. Manjavacas, and F. J. García de Abajo, ACS Nano 6, 1766 (2012).

[30] K. I. Bolotin, K. J. Sikes, Z. Jiang, M. Klima, G. Fudenberg, J. Hone, P. Kim, and H. L. Stormer, Solid State Commun. 146, 351 (2008); E. H. Hwang and S. D. Sarma, Phys. Rev. B 75, 205418 (2007).

[31] H. Yan, T. Low, W. Zhu, Y. Wu, M. Freitag, X. Li, F. Guinea, P. Avouris, and F. Xia, Nat. Photonics 7, 394 (2013).

[32] Properties of Crystalline Silicon, edited by R. Hull (The Institution of Electrical Engineers, London, 1999).

[33] M. Kadic, S. Guenneau, S. Enoch, P. A. Huidobro, L. Martin-Moreno, F. J. Garcia-Vidal, J. Renger, and R. Quidant, Nanophotonics 1, 51 (2012).

[34] J. Schiefele, J. Pedros, F. Sols, F. Calle, and F. Guinea, following Letter, Phys. Rev. Lett. 111, 237405 (2013). 